\title{
Detecting delocalization-localization transitions from full density distributions
}

\author{
Miroslav Hopjan, ${ }^{1}$ Giuliano Orso, ${ }^{2}$ and Fabian Heidrich-Meisner ${ }^{1}$ \\ ${ }^{1}$ Institut für Theoretische Physik, Georg-August-Universität Göttingen, \\ Friedrich-Hund-Platz 1, 3707r Göttingen, Germany \\ ${ }^{2}$ Université de Paris, Laboratoire Matériaux et Phénomènes Quantiques, CNRS, F-75013, Paris, France
}

\begin{abstract}
Characterizing the delocalization transition in closed quantum systems with a many-body localized phase is a key open question in the field of nonequilibrium physics. We exploit that localization of particles as realized in Anderson and standard many-body localization (MBL) implies Fock-space localization in single-particle basis sets characterized by a real-space index. Using a recently introduced quantitative measure for Fock-space localization computed from the density distributions, the occupation distance, we systematically study its scaling behavior across delocalozation transitions and identify critical points from scaling collapses of numerical data. Excellent agreement with literature results is found for the critical disorder strengths of noninteracting fermions, such as the one-dimensional Aubry-André and the three-dimensional Anderson model. We observe a distinctively different scaling behavior in the case of interacting fermions with random disorder consistent with a Kosterlitz-Thouless transition. Finally, we use our measure to extract the transition point as a function of filling for interacting fermions.
\end{abstract}

\section{INTRODUCTION}

Anderson localization [1] generalizes to disordered systems of interacting particles [2,3] leading to manybody localization (MBL). MBL conceptually fits into the framework of thermalization in closed quantum systems [4-7] as a generic exception from eigenstate thermalization [8-12]. The MBL transition is visible in properties of many-body eigenstates at a finite energy density [9], such as area-law entanglement [13-15] and in properties of time-evolved states in global quenches, such as slow logarithmic entanglement entropy growth [16-18] or persistent density inhomogeneities $[19,20]$. Experiments with ultracold atoms [19-28], solid-state spin systems [29, 30], trapped ions [31], and superconducting qubits [32-36] emulated various lattice models with disorder and probed their localization properties [19-35]. Some of the experiments measure eigenstate properties, e.g., the level statistics [31, 33], while most of the efforts address dynamical aspects including the imbalance [19, 20, 22-25, 35], the time-dependent entanglement entropy [26, 31, 32, 34], or $n$-point correlators [27, 28, 30].

There is, however, an ongoing debate on the nature of the ergodic-to-MBL transition and even the very existence of MBL in the field's standard model, namely interacting spinless fermions in one dimension (1D), has been challenged [37-47]. The current proposals for the transition in the thermodynamical limit are: (i) a continuous transition with a power-law scaling of correlations [48, 49], (ii) a transition involving Griffiths regions [50, 51], (iii) Kosterlitz-Thouless (KT) type scaling $[41,52-58]$ as a special case of (ii) [51], or (iv) absence of a true MBL phase in the thermodynamic limit $[37,41-44,46,47]$. While numerical methods play a key role, they are limited with regard to the accessible system sizes $[11,14-17,37-49,56,59-67]$. Therefore, there is a clear need to identify observables for the characterization of localization-delocalization transitions that can be measured in state-of-the-art and future experiments with quantum simulators and are easy to compute numerically as well.

Motivated by these open questions, in this work, we establish the recently introduced occupation distance [67] as a useful quantitative measure for characterizing delocalization-localization transitions. As our main results, we first show that the occupation distance detects localization-delocalization transitions in noninteracting Hamiltonians, including the 1D Aubry-André model (AAM) and the three-dimensional (3D) Anderson model (3AM), finding excellent agreement with literature results for the critical disorder strengths. Second, we report evidence for a KT scaling for interacting spinless fermions in 1D. Third, as an application, we study the dependence of the critical disorder strength on filling in the interacting model.

Given a single-particle basis set $\left|\phi_{\alpha}\right\rangle$ and corresponding creation and annihilation operators $c_{\alpha}^{\dagger}, c_{\alpha}$, the occupation distance is:

$$
\delta n_{\alpha}=\left|n_{\alpha}-\left[n_{\alpha}\right]\right|,
$$

where $\left[n_{\alpha}\right]$ are the closest integer to the occupation $n_{\alpha}=\left\langle\Psi\left|c_{\alpha}^{\dagger} c_{\alpha}\right| \Psi\right\rangle$ in a given many-body state $|\Psi\rangle$. In Anderson and MBL insulators, there is Fock-space localization $[3,63,68,69]$ in the basis of quasiparticles (l-bits) [18, 70-72], the eigenbasis of one-particle densitymatrices [63, 67, 73-78], the Anderson eigenstates [73], and in the basis of physical densities $n_{i}=\left\langle\Psi\left|c_{i}^{\dagger} c_{i}\right| \Psi\right\rangle$ $[67,73]$. Here, we concentrate on the latter, since these objects are the easiest to obtain numerically and experimentally.

To illustrate the concept of our approach, in Fig. 1, we show a typical distribution of $n_{i}$ sampled over eigenstates and disorder realizations for a half-filled chain of interacting spinless fermions [64, 67, 79], whose model is defined in Eq. (3) below. The distribution has a Gaussian-like shape in the delocalized regime [see Fig. 1(a)] and its width shrinks as the system size increases. By contrast, in the localized regime [see Fig. 1(b)], the distribution 

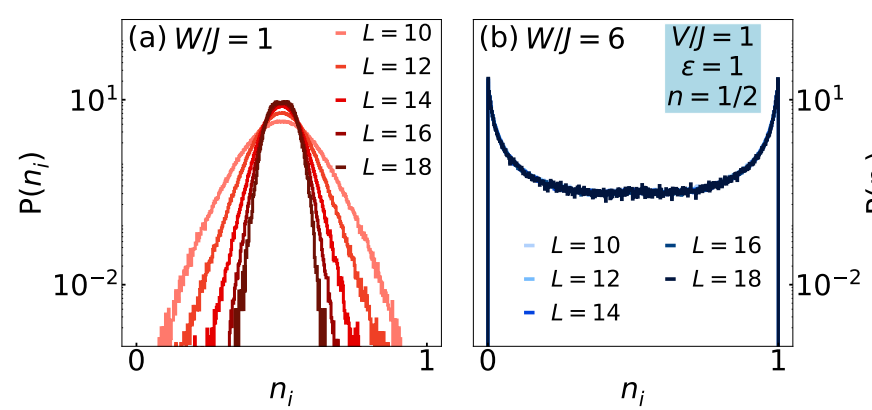

FIG. 1. Interacting fermions, Eq. (3), with $V / J=1, \epsilon=1$, $n=1 / 2$ : Full distributions of local occupations $n_{i}$ in (a) the delocalized $(W / J=1)$ and $(\mathrm{b})$ the localized phase $(W / J=6)$ for $L=10,12,14,16,18$. In (b), the distributions overlap.

has a bimodal structure and is independent of system size, which is typical for the localized phase.

In Fig. 2, we show a typical distributions of $\delta n_{i}$ corresponding to the distrubutions of $n_{i}$ in Fig. 1. The distribution has a maximum at $\delta n_{i}=n=0.5$ in the delocalized regime [see Fig. 2(a)] which gets larger as the system size increases. By contrast, in the localized regime [see Fig. 2(b)], the distribution has a sharp maximum at $\delta n_{i}=0$ and its shape is almost independent of $L$. Hence, in the delocalized regime, the disorder-averaged occupation distance $\overline{\delta n_{i}}$ approaches the average particle filling $n=N / L$ for $N, L \rightarrow \infty$ ( $N$ being the particle number and $L$ the number of sites), while in the localized regime, it saturates to a lower value [67]. In the following, we determine the position of the critical point by studying the scaling properties of $n-\overline{\delta n_{i}}$ on the delocalized side of delocalization-localization transitions.

\section{MODELS}

To establish the validity of our approach, we first apply it to non-interacting systems given by

$$
H=-J \sum_{\langle i j\rangle}\left(c_{i}^{\dagger} c_{j}+h . c\right)+\sum_{i} \epsilon_{i} n_{i}
$$

that exhibit a localization-delocalization transition ( $J$ is the hopping matrix element). For the AAM, the external potential in Eq. (2) is quasi-periodic, $\epsilon_{i}=W \cos (2 \pi q i+$ $\phi)$, where $\phi$ is a global phase and $W$ is the amplitude of the potential which is incommensurate for an irrational wave number $q$. A standard choice for $q$ is the inverse golden ratio $q=\frac{\sqrt{5}-1}{2}$. The AAM has an inherent self-duality at $W_{c} / J=2$ giving rise to a sharp metal-insulator transition [80-90], observed experimentally using cold atoms [91, 92] and photonic lattices [93]. In the $3 \mathrm{AM}$, fermions hop on a $3 \mathrm{D}$ lattice with uncorrelated random on-site energies $\epsilon_{i} \in[-W / 2, W / 2]$. Numerical studies of transport properties [94-97] based on the transfer-matrix technique have shown that, at half filling, the system remains insulating for $W>W_{c} \approx 16.54 \mathrm{~J}$
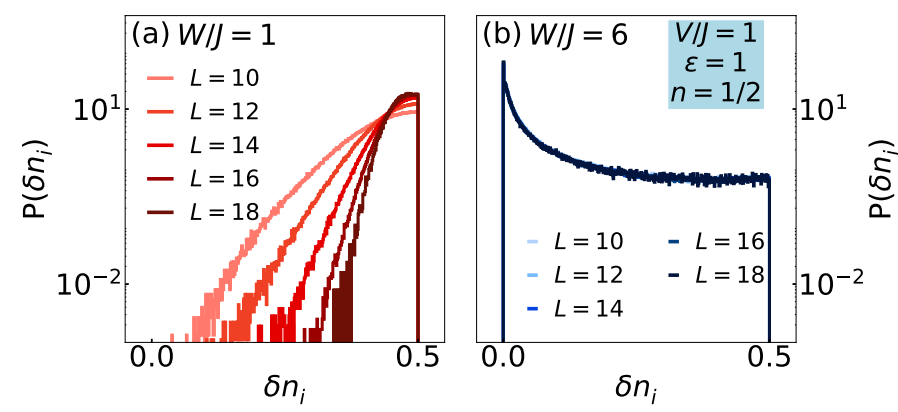

FIG. 2. Interacting fermions, Eq. (3), with $V / J=1, \epsilon=1$, $n=1 / 2$ : Full distributions of the occupation distancies $\delta n_{i}$ (a) in the delocalized phase $(W / J=1)$ and (b) in the localized phase $(W / J=6)$ for $L=10,12,14,16,18$ are shown. For the localized phase in (b), the distributions overlap.

[98] and below $W_{c}$, it is diffusive $[99,100]$. At the transition, the 3AM exhibits subdiffusion [99] and multifractal single-particle wave functions [101, 102].

To investigate the MBL transition, we consider spinless fermions with a nearest-neighbor interaction described by the Hamiltonian

$$
\begin{aligned}
H=\sum_{i=1}^{L}\left[-\frac{J}{2}\left(c_{i}^{\dagger} c_{i+1}+\right.\right. & h . c)+\epsilon_{i}\left(n_{i}-\frac{1}{2}\right) \\
& \left.+V\left(n_{i}-\frac{1}{2}\right)\left(n_{i+1}-\frac{1}{2}\right)\right] .
\end{aligned}
$$

where $c_{i}^{(\dagger)}$ is a fermionic creation/annihilation operator, $n_{i}=c_{i}^{\dagger} c_{i}$ is the density at site $i, J / 2$ is the hopping matrix element, $V$ is the strength of the nearest-neighbor interactions, and $\epsilon_{i}$ is a random potential drawn from a uniform box distribution $[-W, W]$ (we use a different convention for $W$ compared to the $3 \mathrm{AM}$ to be consistent with the MBL literature). Using a Jordan-Wigner transformation, Eq. (3) maps onto a spin-1/2 XXZ chain with random local magnetic field (note the factor $\frac{J}{2}$ in front of the hopping term), a standard system for studies of MBL [12, 48, 60, 61].

We define the target energy density via $\epsilon=\frac{2\left(E-E_{\min }\right)}{E_{\max }-E_{\min }}$, where $E$ is the many-body energy of a particular eigenstate and $E_{\max }$ and $E_{\min }$ are the maximum and minimum energy for each disorder realization, respectively. Hence $\epsilon=1$ corresponds to the middle of the many-body spectrum.

\section{RESULTS FOR NON-INTERACTING MODELS}

We solve the single-particle problem $H \phi_{\alpha}=\epsilon_{\alpha} \phi_{\alpha}$ with periodic boundary conditions using full exact diagonalization for $L \leq 20000$. To obtain those single-particle states $\epsilon_{\alpha}$ contributing to $\epsilon=1$ many-body states, we use a Monte-Carlo generation to sample the statistics of occupied single-particle orbitals. To this end we generate two array $O$ and $U$, containing the indices of occupied 


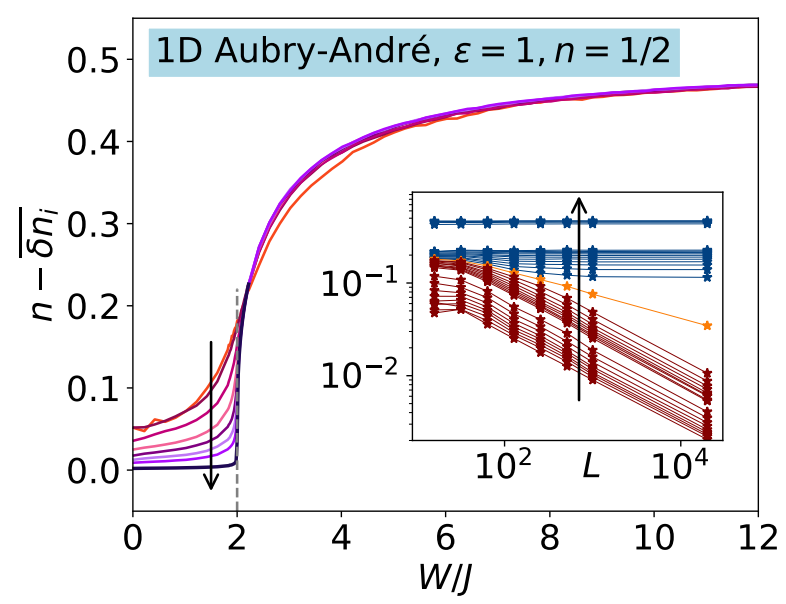

FIG. 3. AAM: $n-\overline{\delta n_{i}}$ as a function of $W / J$ for $L=$ $16,32,64,128,256,512,1000,20000$. The arrow specifies increasing $L$ and the dashed line denotes the transition point [80]. Inset: Log-log plot of the data from the main panel as a function of $L$ deep in the delocalized regime $(W / J=$ $0.02-1.62$, in steps of 0.2 , red $)$, close to the transition $(W / J=$ $1.82-2.22$, in steps of 0.02 , yellow being the transition point), and deep in the localized phase $(W / J=6.02-12.02$, in steps of 2 , blue). The arrow indicates increasing disorder strength.

and unoccupied single-particle states, respectively (for half filling both arrays have length $L / 2$ ). The initialization of these two arrays is random. Once we have the two arrays, we check if the many-body energy $E=\sum_{\alpha \in O} \epsilon_{\alpha}$ belongs to the target energy window. If not, then we generate a new many-body state by taking a random integer from each arrays, $r_{O}$ and $r_{U}$, and we exchange the corresponding elements $O_{r_{O}} \leftrightarrow U_{r_{U}}$. We continue until we find a many-body state with $E$ which belongs to the target energy window. For each disorder realization out of (up to) $10^{4}$ samples, we take one such many-body eigenstate with $\epsilon \in(0.9995,1.0005)$.

In the main panel of Fig. 3, we plot $n-\overline{\delta n_{i}}$ as a function of disorder strength for different system sizes. As expected, in the delocalized phase $(W / J<2)$, this quantity quickly decays to zero for $L \rightarrow \infty$, while in the localized phase $(W / J>2)$, it saturates to a finite value. This saturation is reached for system sizes larger than the localization length. For the largest system sizes, we observe saturation in almost the whole localized phase except for a tiny window around the transition, where the localization length diverges.

To get further insights, in the inset of Fig. 3, we plot $n-\overline{\delta n_{i}}$ as a function of $L$ in $\log$-log scale for different disorder strength $W / J$. For $L>200$, the decay is governed by a power law $L^{-\alpha}$ with $\alpha \approx 0.5$ for all values of $W / J<2$. Remarkably, exactly at the transition point, the decay is governed by a different exponent, $\alpha_{c} \approx 0.25$, revealing the different nature of the single-particle wavefunctions at the critical point [82-90]. In the localized regime, the saturation is reached already for $L \approx 1000$ even for values of $W / J$ close to the transition point. To summarize, for the AAM, there is a remarkable difference

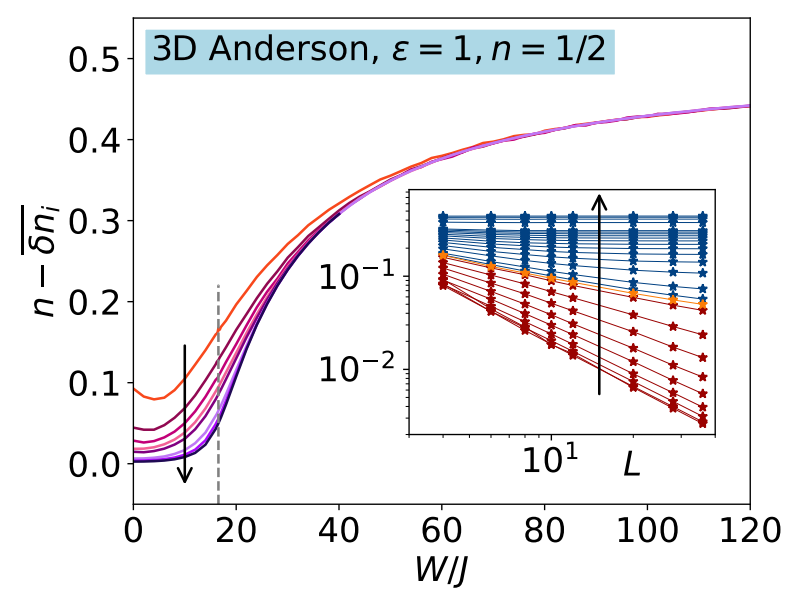

FIG. 4. 3AM, $n-\overline{\delta n_{i}}$ as a function of $W / J$ for $L^{3}=$ $4^{3}, 6^{3}, 8^{3}, 10^{3}, 12^{3}, 20^{3}, 28^{3}, 36^{3}$. The arrow specifies increasing system size and the dashed line denotes the transition point estimated from other measures $W_{c} / J \approx 16.54$ [98]. Inset: Log-log plot of the data from the main panel as a function of $L$ in the delocalized regime $(W / J=0.02-16.02$ in steps of 2 , red), estimated transition point [98] $\left(W_{c} / J \approx 16.54\right.$, yellow) and in the localized phase $(W / J=17.02,18.02-40.02$ in steps of 2 , blue). The arrow specifies increasing disorder strength.

of the behavior of the occupation distance comparing the localized to the delocalized phases. The power-law decay of $n-\overline{\delta n_{i}}$ in the metallic phase and its saturation to a constant in the localized regime is clearly reminiscent of the behavior of the inverse participation ratio in the single-particle problem.

The corresponding analogue of Fig. 3 for the 3AM is displayed in Fig. 4. Here, $n-\overline{\delta n_{i}}$ clearly decays to zero for $L \rightarrow \infty$ in the delocalized phase $(W / J<16)$. Oppositely, in the localized regime, there is a clear onset of saturation for $L \approx 20-36$ for $W / J>17$. The trends are less clear for $W_{c} / J \approx 16-17$, i.e., close to the estimated transition $W_{c} / J \approx 16.54$ [98]. We observe that for all $L$ values considered here, the decay in the delocalized phase is governed by a power law $L^{-\alpha}$, but the exponent $\alpha$ now depends on the disorder strength, most probably due to the limited system sizes available. At the estimated transition point [98], we find that the decay is governed by the exponent $\alpha_{c} \approx 0.75(5)$.

We next perform a scaling collapse of the data of Fig. 3, focusing on the delocalized phase and the vicinity of the transition point. For this purpose, in Fig. 5, we replot the data using the dimensionless variables $w=L^{1 / \nu}\left(W-W_{c}\right) / J$ and $\left(n-\overline{\delta n_{i}}\right) L^{\mu}$ for the $x$ and $y$ axis, respectively. To get the best-quality estimate of the exponent $\nu$, the scaling collapse needs to be performed for system sizes in the scaling regime. Therefore, we include only systems with $L \geq 256$, which is a posteriori justified by the results, see the inset of Fig. 3. For the AAM, we then find $\mu \simeq 0.25(5), W_{c} / J=2.00(5)$ and the expected critical exponent $\nu \simeq 1.05(5)$, see the main 


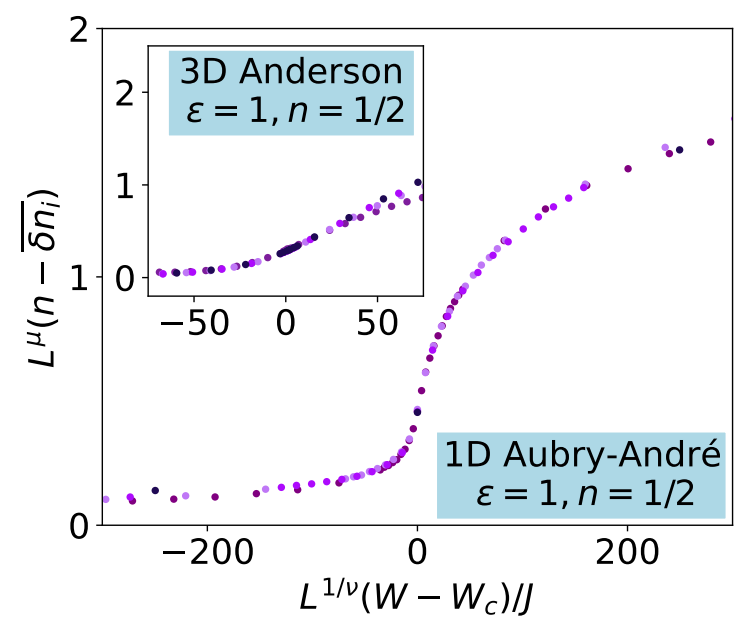

FIG. 5. AAM: Scaling collapse of $n-\overline{\delta n_{i}}$ for $L=256,512,1000,20000$ and fitting parameters $W_{c}=$ 2.00(5) $J, \mu=0.25(5), \nu=1.05(5)$. Inset: Scaling collapse for the 3AM using $L^{3}=12^{3}, 20^{3}, 28^{3}, 36^{3}$ with fitting parameters $W_{c}=16.3(2) J, \mu=0.50(5), \nu=1.60(5)$.

panel of Fig. 5. As a side remark, note that $\mu \simeq \alpha_{c}$, so that the product $\left(n-\overline{\delta n_{i}}\right) L^{\mu}$ takes a finite value at the transition point. Interestingly, we have recovered the values of $W_{c} / J=2$ and $\nu=1$ known from the single-particle transition [80,81], which suggests that the Fock-space delocalization-localization transition is driven by the divergent localization (correlation) length in the single-particle states.

We carry out the same scaling-collapse procedure for the 3AM. Here, the best up-to-date estimate for the critical disorder strength is $W_{c} / J \approx 16.54$ [98], which is obtained by the transfer matrix method for the midspectrum states of the single-particle Hamiltonian. In the inset of Fig. 5, we show the scaling collapse, obtained by retaining only the data sets with $L \geq 12$, see the inset of Fig. 4. We then find $\mu \simeq 0.50(5), W_{c} / J=16.3(2)$ and the expected critical exponent $\nu \simeq 1.60(5)$, see the inset of Fig. 5. The estimates of the critical point $W_{c} / J \simeq 16.3(2)$ and the critical exponent $\nu \simeq 1.60(5)$ are quite close to the values $W_{c} / J=16.54, \nu=1.572 \mathrm{ob}-$ tained by the transfer-matrix method [98], where much larger system sizes (up to $L=64$ ) were considered. We attribute the small differences between the estimates to residual finite-size effects. Additionally, the manybody Slater determinant can contain a fraction of singleparticle states whose energy lies close to the band edge. Since these states localize for a much weaker disorder, it is in principle possible that $n-\overline{\delta n_{i}}$ saturates to a small but finite value in the vicinity of the critical point, in contrast to the scaling ansatz. For the system sizes that we considered, however, we do not find clear evidence for this effect.

The presented results for the AAM and the 3AM confirm that the occupation distance accurately captures the transitions well. Thus, remarkably, by exploiting Fockspace localization, the transition points can be deter-

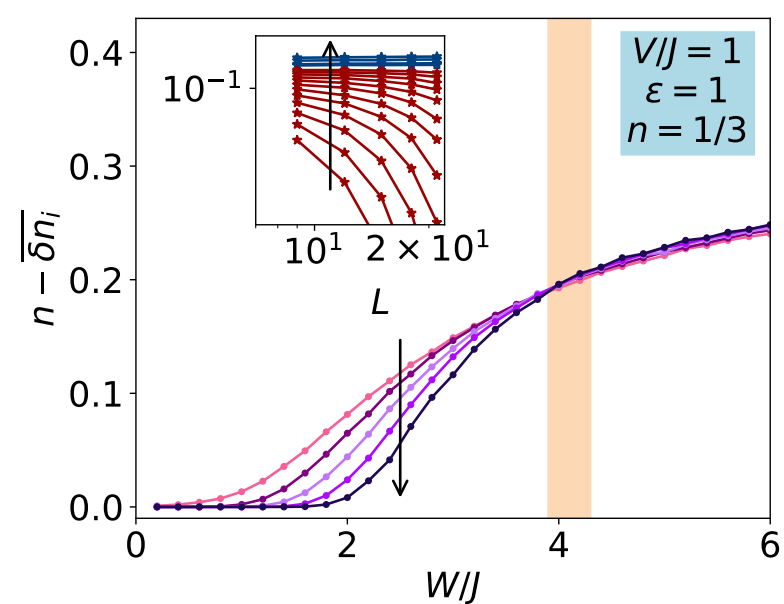

FIG. 6. 1D interacting fermions, Eq. (3), $(\epsilon=1, V / J=1, n=$ 1/3): $n-\overline{\delta n_{i}}$ as a function of $W / J$ for $L=9,12,15,18,21$. The arrow specifies increasing system size and the shaded area indicates the estimated range of the transition point. Inset: Log-log plot of the data from the main panel as a function of $L$ in the delocalized regime (red symbols, $W / J=1.2-3.6$, in steps of 0.2 ) and in the localized phase (blue symbols, $W / J=$ $4.2,5.0,6.0)$. The arrow specifies increasing disorder strength.

mined from the distributions of the simple-to-calculate quantity $n_{i}$. Moreover, the advances with characterizing MBL also feedback into devising hitherto unexplored approaches for disordered non-interacting models (see also [103-105]).

\section{RESULTS FOR INTERACTING MODELS}

We now turn our attention to the interacting model in Eq. (3), considering values of the filling $1 / 10 \leq n \leq 2 / 3$, $L \leq 30$, and $10^{4}$ disorder realizations (see the appendix for details). We impose periodic boundary conditions. For a given disorder realization, we use the shift-andinvert method $[48,106]$ to efficiently extract the six eigenstates closest to the target energy $\epsilon=1$.

The main panel of Fig. 6 shows $n-\overline{\delta n_{i}}$ as a function of disorder strength for $n=1 / 3$ (for other values of $n$, the behavior is similar). For $W / J<3.6$, there is a clear decay towards zero while for $W / J>4.2$, there is a slow saturation to a finite value. However, the decay in the delocalized phase has a different character from the one previously observed for the non-interacting models. In particular, see the inset of Fig. 6, the decay of the average occupation distance is not algebraic, but exponential. This is a consequence of the exponential decay of the fluctuations according to the eigenstate thermalisation hypothesis [4-6, 107].

The exponential dependence on $L$ in the delocalized phase suggests that, for the interacting system, the scaling collapse of the data in Fig. 6 is of the KT type. We also add that the KT form of the scaling is inspired by the recent literature on the phenomelogical renormalisation 


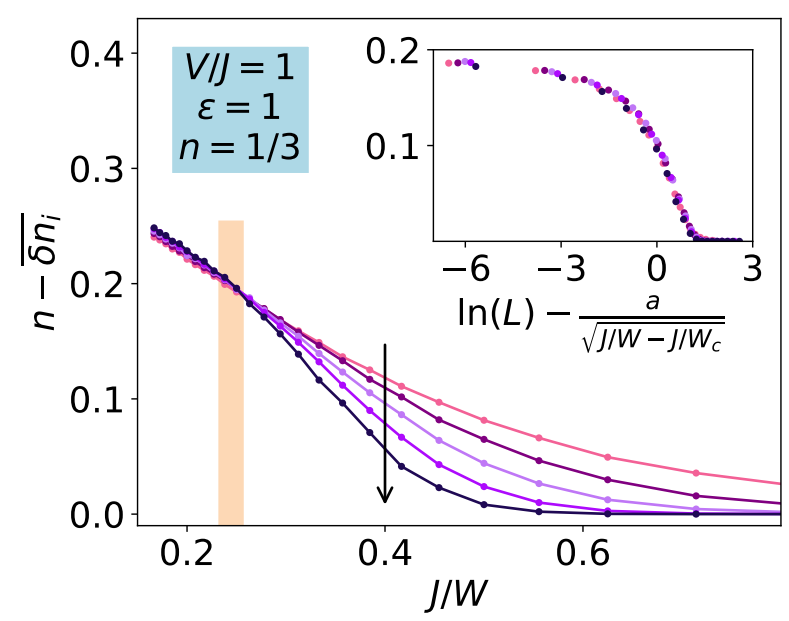

FIG. 7. 1D interacting fermions, Eq. (3), with $\epsilon=1, V / J=$ $1, n=1 / 3: \quad n-\overline{\delta n_{i}}$ as a function of $J / W$ for $L=$ $9,12,15,18,21$. The arrow specifies increasing system size and the shaded area indicates the estimated range of the transition point. Inset: Scaling collapse of the data from the main panel in the delocalized phase as a function of $\ln (L / \xi)$ where $\xi=\exp \left(-a / \sqrt{J / W-J / W_{c}}\right)$ with fitting parameters $W_{c} / J=4.1(2)$ and $a=1.00(5)$. For the scaling collapse all the data in the ergodic regime, i.e. with $J / W>J / W_{c}$, are used.

group theories of the MBL transitions [52-54]. To verify this hypothesis, in Fig. 7 (main panel), we first display $n-\overline{\delta n_{i}}$ as a function of $J / W$. Then, in the inset of the same figure, we plot the same data as a function of the variable $\ln (L / \xi)$, where $\xi=\exp \left(-a / \sqrt{J / W-J / W_{c}}\right)$ is the KT length, with $W_{c}$ and $a$ being fitting parameters. Evidently, a KT-like scaling for the occupation distance is consistent with our data and the collapse is of similar quality in the whole delocalised region. We note that with the power-law scaling, we did not obtain any satisfactory collapse of our data (not shown). Interestingly, a KT scaling predicts a jump of the occupation distance at the critical point $W_{c}$ for $L \rightarrow \infty$, which agrees with the jump of the multi-fractal dimension of the eigenstates at the localization-delocalization transition reported in $[58,66,108,109]$. Our analysis of full density distributions is advantageous over the study of the minimum deviation from $n_{i}=1-$ a single number - studied in [56], since our measure suffers less from numerical or experimental uncertainties.

Finally, we repeat the same procedure for different particle fillings to extract $W_{c}=W_{c}(n)$. The full density dependence of the transition point in the interacting system was not explored before and is an additional quantitative prediction which could be explored in future experiments. The density dependence of the transition in the interacting system is different from the AAM where the critical disorder strength is the same for all fillings, thus the $n$-dependence of $W_{c} / J$ hints at a different mechanism of the transition in interacting systems. The resulting phase boundary between MBL and delocalized states is displayed in Fig. 8. First, note that the critical

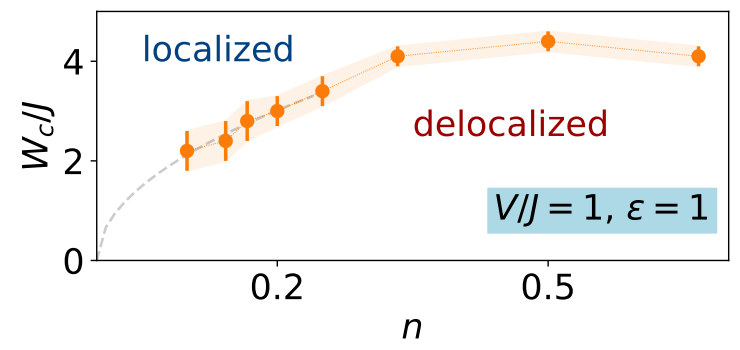

FIG. 8. 1D interacting fermions Eq. (3), with $\epsilon=1, V / J=$ 1: Transition point $W_{c} / J$ as a function of filling $n$ (dots) estimated from scaling collapses as in Fig. 7. The dashed line is a power-law fit to $W_{c} \approx c \cdot n^{\lambda}$ where $\lambda=0.58(10)$.

disorder strength is particle-hole symmetric with respect to filling, i.e., $W_{c}(n)=W_{c}(1-n)$. Second, $W_{c}$ takes its maximum value at half-filling, where $W_{c} / J=4.4(2)$; consistent with other works $[48,56,63,66,67]$ (see also [37-41, 49, 110-114]). Third, for $n<0.5, W_{c} / J$ decreases steadily as the filling diminishes and must vanish at zero filling. Indeed, all single-particle states are localized in an infinite lattice and few-particle states remain also localized, although short-range interactions can substantially increase their localization length [115-117] (a similar situation occurs also in two dimensions, see [118]). By fitting the data in Fig. 8 at low fillings with a power law $W_{c} / J \approx c \cdot n^{\lambda}$, where $c$ and $\lambda$ are fitting parameters, we obtain $\lambda=0.58(10)$.

\section{CONCLUSIONS}

We demonstrated that the quantitative analysis of density distributions is instrumental for the characterization of localization-delocalization transitions. Our approach based on the occupation distance exploits both real-space and Fock-space localization as a characteristic properties of states with localized (quasi-)particles. We showed that the average occupation distance [67], extracted from the density distributions, exhibits critical scaling behavior at the transition of noninteracting models such as the 1D Aubry-André and the 3D Anderson model as well as of interacting spinless fermions. In the noninteracting models, the average occupation distance collapses with a power-law decay while in the interacting model, the observed KT scaling hints at a different mechanism of the MBL transition consistent with predictions of $[41,52-$ $54,56,57]$. Finally, we extract the filling dependence of the transition point and observe an approximate squareroot dependence $W_{c} \propto \sqrt{n}$.

The measurement of densities is simple and suffers less from errors in numerical methods such as DMRG than more complicated observables or time-dependent objects [119]. Regarding quantum-gas experiments, while these do not access eigenstates, the measurement of distributions of densities after slow loading processes into disorder potentials may yield similar information, 


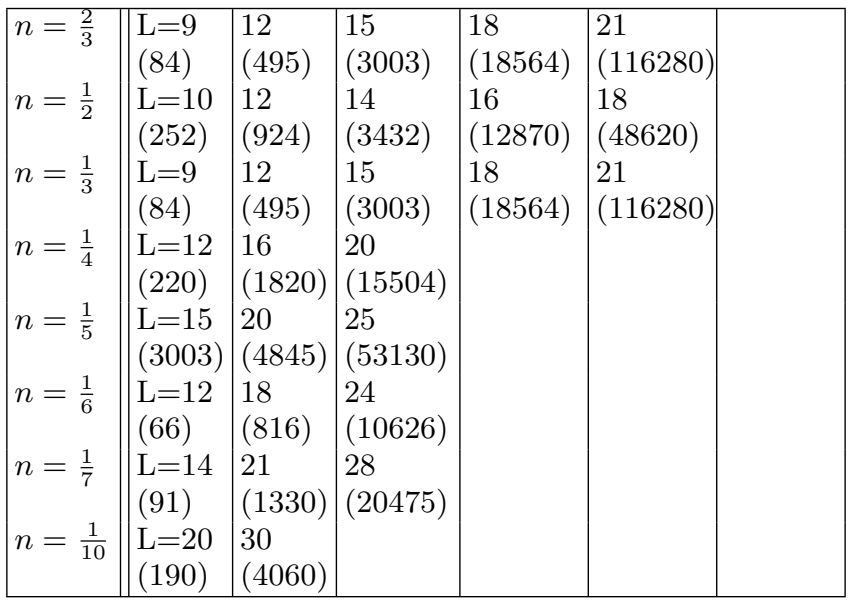

TABLE I. System sizes for the interacting system considered in this study, the corresponding sizes of the Hilbert space are written in the brackets.

to be studied further. Such experiments would neither be restricted to small systems as is the case for the measurment of the entanglement entropy $[26,34]$ nor require time-dependent measurements, further reducing uncertainties. Our results thus provide a path for studying delocalization-localization transitions in future scaled-up quantum-gas microscope experiments, aiming at clarifying the nature of the transition in interacting systems and the existence of the MBL phase.

\section{ACKNOWLEDGMENTS}

We thank J. Bardarson and L. Vidmar for useful comments on an earlier version of the manuscript and for helpful discussions.

\section{Appendix: Finite-size scaling ansatz and data collapses}

We now turn to the scaling collapses of $n-\overline{\delta n_{i}}$. For the non-interacting models, i.e., the AAM and the $3 \mathrm{AM}$, we consider the scaling ansatz of the form $L^{-\mu} f\left(w L^{1 / \nu}\right)$ where $w=W / J-W_{c} / J$ is the distance from the transition point, $f(t)$ is the scaling function of interest, and $\left\{W_{c}, \mu, \nu\right\}$ are the fitting parameters. In the interacting model, we use an ansatz of the KT form $g\left(\ln (L)-\ln \left(a / \sqrt{J / W-J / W_{c}}\right)\right)$ with $g(t)$ being the scaling function of interest and $\left\{W_{c}, a\right\}$ are the fitting parameters. The system sizes considered for the interacting model are listed in Table I. We calculate 6 eigenstates close to $\epsilon=1 / 2$ for $10^{4}$ disorder realization for Hilbert spaces $<25000$ and $10^{2}-10^{3}$ disorder realization for the larger ones. Note that this gives in total $L \cdot 6 \cdot 10^{4}$ and $L \cdot 6 \cdot\left(10^{2}-10^{3}\right)$ values of the density $n_{i}$ for the density histograms which is sufficient for converged results for the averages.

To assess the quality of the scaling collapses, i.e., the smoothness of the functions $f(t)$ and $g(t)$ of the argument $t$, we use a cost function introduced in Ref. [41]:

$$
\mathcal{C}_{X}=\frac{\sum_{j=1}^{N_{p}-1}\left|X_{j+1}-X_{j}\right|}{\max \left\{X_{j}\right\}-\min \left\{X_{j}\right\}}-1
$$

where $X$ stands for the values of $f$ and $g$ and the set $\left\{X_{j}\right\}$ of $N_{p}$ values $X_{j}$ is ordered by the values of the argument $t$.
[1] P. W. Anderson, Phys. Rev. 109, 1492 (1958).

[2] I. V. Gornyi, A. D. Mirlin, and D. G. Polyakov, Phys. Rev. Lett. 95, 206603 (2005).

[3] D. Basko, I. Aleiner, and B. Altshuler, Ann. Phys. (N.Y.) 321, 1126 (2006).

[4] J. M. Deutsch, Phys. Rev. A 43, 2046 (1991).

[5] M. Srednicki, Phys. Rev. E 50, 888 (1994).

[6] M. Rigol, V. Dunjko, and M. Olshanii, Nature 452, 854 (2008).

[7] L. D'Alessio, Y. Kafri, A. Polkovnikov, and M. Rigol, Adv. Phys. 65, 239 (2016).

[8] E. Altman and R. Vosk, Ann. Rev. Cond. Matt. Phys. 6, 383 (2015).

[9] R. Nandkishore and D. A. Huse, Ann. Rev. Cond. Matt. Phys. 6, 15 (2015).

[10] E. Altman, Nat. Phys. 14, 979 (2018).

[11] F. Alet and N. Laflorencie, C. R. Phys. 19, 498 (2018).

[12] D. A. Abanin, E. Altman, I. Bloch, and M. Serbyn, Rev. Mod. Phys. 91, 021001 (2019).

[13] B. Bauer and C. Nayak, J. Stat. Mech. Theor. Exp.
2013, P09005 (2013).

[14] J. A. Kjäll, J. H. Bardarson, and F. Pollmann, Phys. Rev. Lett. 113, 107204 (2014).

[15] M. Friesdorf, A. H. Werner, W. Brown, V. B. Scholz, and J. Eisert, Phys. Rev. Lett. 114, 170505 (2015).

[16] M. Žnidarič, T. Prosen, and P. Prelovšek, Phys. Rev. B 77, 064426 (2008).

[17] J. H. Bardarson, F. Pollmann, and J. E. Moore, Phys. Rev. Lett. 109, 017202 (2012).

[18] M. Serbyn, Z. Papić, and D. A. Abanin, Phys. Rev. Lett. 110, 260601 (2013).

[19] M. Schreiber, S. S. Hodgman, P. Bordia, H. P. Lüschen, M. H. Fischer, R. Vosk, E. Altman, U. Schneider, and I. Bloch, Science 349, 842 (2015).

[20] J.-Y. Choi, S. Hild, J. Zeiher, P. Schauß, A. RubioAbadal, T. Yefsah, V. Khemani, D. A. Huse, I. Bloch, and C. Gross, Science 352, 1547 (2016).

[21] S. S. Kondov, W. R. McGehee, W. Xu, and B. DeMarco, Phys. Rev. Lett. 114, 083002 (2015).

[22] H. P. Lüschen, P. Bordia, S. Scherg, F. Alet, E. Alt- 
man, U. Schneider, and I. Bloch, Phys. Rev. Lett. 119 260401 (2017).

[23] P. Bordia, H. Lüschen, S. Scherg, S. Gopalakrishnan, M. Knap, U. Schneider, and I. Bloch, Phys. Rev. X 7, 041047 (2017).

[24] T. Kohlert, S. Scherg, X. Li, H. P. Lüschen, S. Das Sarma, I. Bloch, and M. Aidelsburger, Phys. Rev. Lett. 122, 170403 (2019).

[25] A. Rubio-Abadal, J.-Y. Choi, J. Zeiher, S. Hollerith, J. Rui, I. Bloch, and C. Gross, Phys. Rev. X 9, 041014 (2019).

[26] A. Lukin, M. Rispoli, R. Schittko, M. E. Tai, A. M. Kaufman, S. Choi, V. Khemani, J. Léonard, and M. Greiner, Science 364, 256 (2019).

[27] M. Rispoli, A. Lukin, R. Schittko, S. Kim, M. E. Tai, J. Léonard, and M. Greiner, Nature 573, 385 (2019).

[28] J. Léonard, M. Rispoli, S. R. Lukin, A., S. Kim, J. Kwan, D. Sels, E. Demler, and M. Greiner, arXiv:2012.15270.

[29] G. A. Álvarez, D. Suter, and R. Kaiser, Science 349, 846 (2015).

[30] K. X. Wei, C. Ramanathan, and P. Cappellaro, Phys. Rev. Lett. 120, 070501 (2018)

[31] J. Smith, A. Lee, P. Richerme, B. Neyenhuis, P. W. Hess, P. Hauke, M. Heyl, D. A. Huse, and C. Monroe, Nat. Phys. 12, 907 (2016).

[32] K. Xu, J.-J. Chen, Y. Zeng, Y.-R. Zhang, C. Song, W. Liu, Q. Guo, P. Zhang, D. Xu, H. Deng, K. Huang, H. Wang, X. Zhu, D. Zheng, and H. Fan, Phys. Rev. Lett. 120, 050507 (2018).

[33] P. Roushan, C. Neill, J. Tangpanitanon, V. M. Bastidas, A. Megrant, R. Barends, Y. Chen, Z. Chen, B. Chiaro, A. Dunsworth, A. Fowler, B. Foxen, M. Giustina, E. Jeffrey, J. Kelly, E. Lucero, J. Mutus, M. Neeley, C. Quintana, D. Sank, A. Vainsencher, J. Wenner, T. White, H. Neven, D. G. Angelakis, and J. Martinis, Science 358, 1175 (2017).

[34] B. Chiaro, C. Neill, A. Bohrdt, M. Filippone, F. Arute, K. Arya, R. Babbush, D. Bacon, J. Bardin, R. Barends, S. Boixo, D. Buell, B. Burkett, Y. Chen, Z. Chen, R. Collins, A. Dunsworth, E. Farhi, A. Fowler, B. Foxen, C. Gidney, M. Giustina, M. Harrigan, T. Huang, S. Isakov, E. Jeffrey, Z. Jiang, D. Kafri, K. Kechedzhi, J. Kelly, P. Klimov, A. Korotkov, F. Kostritsa, D. Landhuis, E. Lucero, J. McClean, X. Mi, A. Megrant, M. Mohseni, J. Mutus, M. McEwen, O. Naaman, M. Neeley, M. Niu, A. Petukhov, C. Quintana, N. Rubin, D. Sank, K. Satzinger, A. Vainsencher, T. White, Z. Yao, P. Yeh, A. Zalcman, V. Smelyanskiy, H. Neven, S. Gopalakrishnan, D. Abanin, M. Knap, J. Martinis, and P. Roushan, arXiv:1910.06024.

[35] Q. Guo, C. Cheng, Z.-H. Sun, Z. Song, H. Li, Z. Wang, W. Ren, H. Dong, D. Zheng, Y.-R. Zhang, R. Mondaini, H. Fan, and H. Wang, Nature Physics 17, 234 (2021).

[36] M. Gong, G. D. de Moraes Neto, C. Zha, Y. Wu, H. Rong, Y. Ye, S. Li, Q. Zhu, S. Wang, Y. Zhao, F. Liang, J. Lin, Y. Xu, C.-Z. Peng, H. Deng, A. Bayat, X. Zhu, and J.-W. Pan, Phys. Rev. Research 3, 033043 (2021).

[37] J. Šuntajs, J. Bonča, T. Prosen, and L. Vidmar, Phys. Rev. E 102, 062144 (2020).

[38] P. Sierant, D. Delande, and J. Zakrzewski, Phys. Rev. Lett. 124, 186601 (2020).

[39] D. Abanin, J. Bardarson, G. De Tomasi, S. Gopalakr- ishnan, V. Khemani, S. Parameswaran, F. Pollmann, A. Potter, M. Serbyn, and R. Vasseur, Annals of Physics 427, 168415 (2021).

[40] R. K. Panda, A. Scardicchio, M. Schulz, S. R. Taylor, and M. Žnidarič, EPL 128, 67003 (2019).

[41] J. Šuntajs, J. Bonča, T. Prosen, and L. Vidmar, Phys. Rev. B 102, 064207 (2020).

[42] D. Sels and A. Polkovnikov, Phys. Rev. E 104, 054105 (2021).

[43] T. LeBlond, D. Sels, A. Polkovnikov, and M. Rigol, arXiv:2012.07849.

[44] M. Kiefer-Emmanouilidis, R. Unanyan, M. Fleischhauer, and J. Sirker, Phys. Rev. Lett. 124, 243601 (2020).

[45] D. J. Luitz and Y. B. Lev, Phys. Rev. B 102, 100202 (2020).

[46] M. Kiefer-Emmanouilidis, R. Unanyan, M. Fleischhauer, and J. Sirker, Annals of Physics , 168481 (2021).

[47] M. Kiefer-Emmanouilidis, R. Unanyan, M. Fleischhauer, and J. Sirker, Phys. Rev. B 103, 024203 (2021).

[48] D. J. Luitz, N. Laflorencie, and F. Alet, Phys. Rev. B 91, 081103 (2015).

[49] V. Khemani, D. N. Sheng, and D. A. Huse, Phys. Rev. Lett. 119, 075702 (2017).

[50] K. Agarwal, S. Gopalakrishnan, M. Knap, M. Müller, and E. Demler, Phys. Rev. Lett. 114, 160401 (2015).

[51] Z. Lenarčič, O. Alberton, A. Rosch, and E. Altman, Phys. Rev. Lett. 125, 116601 (2020).

[52] A. Goremykina, R. Vasseur, and M. Serbyn, Phys. Rev. Lett. 122, 040601 (2019).

[53] P. T. Dumitrescu, A. Goremykina, S. A. Parameswaran, M. Serbyn, and R. Vasseur, Phys. Rev. B 99, 094205 (2019).

[54] A. Morningstar and D. A. Huse, Phys. Rev. B 99, 224205 (2019).

[55] L. Herviou, S. Bera, and J. H. Bardarson, Phys. Rev. B 99, 134205 (2019).

[56] N. Laflorencie, G. Lemarié, and N. Macé, Phys. Rev. Research 2, 042033 (2020).

[57] A. Morningstar, D. A. Huse, and J. Z. Imbrie, Phys. Rev. B 102, 125134 (2020).

[58] G. De Tomasi, I. M. Khaymovich, F. Pollmann, and S. Warzel, Phys. Rev. B 104, 024202 (2021).

[59] L. F. Santos, J. Phys. A 37, 4723 (2004).

[60] V. Oganesyan and D. A. Huse, Phys. Rev. B 75, 155111 (2007).

[61] A. Pal and D. A. Huse, Phys. Rev. B 82, 174411 (2010).

[62] Y. Bar Lev, G. Cohen, and D. R. Reichman, Phys. Rev. Lett. 114, 100601 (2015).

[63] S. Bera, H. Schomerus, F. Heidrich-Meisner, and J. H. Bardarson, Phys. Rev. Lett. 115, 046603 (2015).

[64] S. P. Lim and D. N. Sheng, Phys. Rev. B 94, 045111 (2016).

[65] V. Khemani, F. Pollmann, and S. L. Sondhi, Phys. Rev. Lett. 116, 247204 (2016).

[66] N. Macé, F. Alet, and N. Laflorencie, Phys. Rev. Lett. 123, 180601 (2019).

[67] M. Hopjan and F. Heidrich-Meisner, Phys. Rev. A 101, 063617 (2020).

[68] S. Roy, J. T. Chalker, and D. E. Logan, Phys. Rev. B 99, 104206 (2019).

[69] D. E. Logan and S. Welsh, Phys. Rev. B 99, 045131 
(2019).

[70] D. A. Huse, R. Nandkishore, and V. Oganesyan, Phys. Rev. B 90, 174202 (2014).

[71] J. Z. Imbrie, V. Ros, and A. Scardicchio, Ann. Phys. (Leipzig) 529, 1600278 (2017).

[72] J. Z. Imbrie, Phys. Rev. Lett. 117, 027201 (2016).

[73] S. Bera, T. Martynec, H. Schomerus, F. HeidrichMeisner, and J. H. Bardarson, Ann. Phys. (Leipzig) 529, 1600356 (2017).

[74] T. L. M. Lezama, S. Bera, H. Schomerus, F. HeidrichMeisner, and J. H. Bardarson, Phys. Rev. B 96, 060202 (2017).

[75] S.-H. Lin, B. Sbierski, F. Dorfner, C. Karrasch, and F. Heidrich-Meisner, SciPost Phys. 4, 002 (2018).

[76] W. Buijsman, V. Gritsev, and V. Cheianov, SciPost Phys. 4, 38 (2018).

[77] B. Villalonga, X. Yu, D. J. Luitz, and B. K. Clark, Phys. Rev. B 97, 104406 (2018).

[78] C. P. Chen, M. Szyniszewski, and H. Schomerus, Phys. Rev. Research 2, 023118 (2020).

[79] D. J. Luitz, Phys. Rev. B 93, 134201 (2016).

[80] S. Aubry and G. André, Ann. Isr. Phys. Soc. 3, 133 (1980).

[81] I. Suslov, JETP 56, 612 (1982).

[82] M. Kohmoto, Phys. Rev. Lett. 51, 1198 (1983).

[83] C. Tang and M. Kohmoto, Phys. Rev. B 34, 2041 (1986).

[84] M. Kohmoto, B. Sutherland, and C. Tang, Phys. Rev. B 35, 1020 (1987).

[85] A. P. Siebesma and L. Pietronero, 4, 597 (1987).

[86] H. Hiramoto and M. Kohmoto, Phys. Rev. B 40, 8225 (1989).

[87] H. Hiramoto and M. Kohmoto, International Journal of Modern Physics B 06, 281 (1992), https://doi.org/10.1142/S0217979292000153.

[88] E. Maciá, ISRN Condensed Matter Physics 2014, 165943 (2014).

[89] X. Li, J. H. Pixley, D.-L. Deng, S. Ganeshan, and S. Das Sarma, Phys. Rev. B 93, 184204 (2016).

[90] A.-K. Wu, "Fractal spectrum of the aubry-andre model," (2021), arXiv:2109.07062 [cond-mat.dis-nn].

[91] G. Roati, C. D'Errico, L. Fallani, M. Fattori, C. Fort, M. Zaccanti, G. Modugno, M. Modugno, and M. Inguscio, Nature 453, 895 (2008).

[92] H. P. Lüschen, S. Scherg, T. Kohlert, M. Schreiber, P. Bordia, X. Li, S. Das Sarma, and I. Bloch, Phys. Rev. Lett. 120, 160404 (2018).

[93] Y. Lahini, R. Pugatch, F. Pozzi, M. Sorel, R. Morandotti, N. Davidson, and Y. Silberberg, Phys. Rev. Lett. 103, 013901 (2009).

[94] B. Kramer and A. MacKinnon, Reports on Progress in Physics 56, 1469 (1993).
[95] B. Kramer, K. Broderix, A. Mackinnon, and M. Schreiber, Physica A: Statistical Mechanics and its Applications 167, 163 (1990).

[96] A. MacKinnon and B. Kramer, Zeitschrift für Physik B Condensed Matter 53, 1 (1983).

[97] A. MacKinnon and B. Kramer, Phys. Rev. Lett. 47, 1546 (1981).

[98] K. Slevin and T. Ohtsuki, J. Phys. Soc. Jpn. 87, 094703 (2018).

[99] T. Ohtsuki and T. Kawarabayashi, J. Phys. Soc. Jpn. 66, 314 (1997).

[100] Y. Zhao, D. Feng, Y. Hu, S. Guo, and J. Sirker, Phys. Rev. B 102, 195132 (2020).

[101] A. Rodriguez, L. J. Vasquez, and R. A. Römer, Phys. Rev. Lett. 102, 106406 (2009).

[102] A. Rodriguez, L. J. Vasquez, K. Slevin, and R. A. Römer, Phys. Rev. Lett. 105, 046403 (2010).

[103] M. J. Gullans and D. A. Huse, Phys. Rev. Lett. 123, 110601 (2019).

[104] J. Šuntajs, T. Prosen, and L. Vidmar, Annals of Physics , 168469 (2021).

[105] P. Prelovšek and J. Herbrych, Phys. Rev. B 103 , L241107 (2021).

[106] F. Pietracaprina, N. Macé, D. J. Luitz, and F. Alet, SciPost Phys. 5, 45 (2018).

[107] W. Beugeling, R. Moessner, and M. Haque, Phys. Rev. E 89, 042112 (2014).

[108] A. Solórzano, L. F. Santos, and E. J. Torres-Herrera, Phys. Rev. Research 3, L032030 (2021).

[109] S. Roy and D. E. Logan, Phys. Rev. B 104, 174201 (2021).

[110] T. Devakul and R. R. P. Singh, Phys. Rev. Lett. 115, 187201 (2015).

[111] E. V. H. Doggen, F. Schindler, K. S. Tikhonov, A. D. Mirlin, T. Neupert, D. G. Polyakov, and I. V. Gornyi, Phys. Rev. B 98, 174202 (2018).

[112] T. Chanda, P. Sierant, and J. Zakrzewski, Phys. Rev. B 101, 035148 (2020).

[113] T. Chanda, P. Sierant, and J. Zakrzewski, Phys. Rev. Research 2, 032045 (2020).

[114] P. Sierant, M. Lewenstein, and J. Zakrzewski, Phys. Rev. Lett. 125, 156601 (2020).

[115] D. L. Shepelyansky, Phys. Rev. Lett. 73, 2607 (1994).

[116] F. von Oppen, T. Wettig, and J. Müller, Phys. Rev. Lett. 76, 491 (1996).

[117] K. M. Frahm, Eur. Phys. J. B 89, 115 (2016).

[118] F. Stellin and G. Orso, Phys. Rev. B 102, 144201 (2020).

[119] U. Schollwöck, Ann. Phys. (N.Y.) 326, 96 (2011). 